Korean J. Math. 21 (2013), No. 2, pp. 181-187

http://dx.doi.org/10.11568/kjm.2013.21.2.181

\title{
A HOMOMORPHISM OF POINTED MINIMAL SETS AND QUASI-ELLIS GROUPS
}

\author{
HYUNG-SOO SONG
}

\begin{abstract}
In this paper we give some results on homomorphisms of pointed minimal sets. In particular, we investigate some characterizations on quasi-Ellis groups.
\end{abstract}

\section{Introduction}

Universal minimal sets were studied by R. Ellis in [2]. In [4], S. Glasner introduced Ellis groups which are certain groups of the universal minimal set. Given a homomorphism of pointed minimal sets $\pi:\left(X, x_{0}\right) \rightarrow\left(Y, y_{0}\right)$, we can define Ellis groups $\mathcal{G}\left(X, x_{0}\right)$ and $\mathcal{G}\left(Y, y_{0}\right)$ and give relationships between the homomorphism and Ellis groups.

In this paper we introduce the concept of quasi-Ellis groups $\mathcal{S}\left(X, x_{0}\right)$ and $\mathcal{S}\left(Y, y_{0}\right)$ which are generalizations of Ellis groups and investigate some characterizations on quasi-Ellis groups.

\section{Preliminaries}

A transformation group, or flow, $(X, T)$, will consist of a jointly continuous action of the topological group $T$ on the compact Hausdorff space

Received March 22, 2013. Revised May 30, 2013. Accepted June 5, 2013.

2010 Mathematics Subject Classification: 54H20.

Key words and phrases: proximal homomorphism, distal homomorphism, regular homomorphism, pointed minimal set, Ellis group, quasi-Ellis group.

The present research has been conducted by the Research Grant of Kwangwoon University in 2012.

(c) The Kangwon-Kyungki Mathematical Society, 2013.

This is an Open Access article distributed under the terms of the Creative commons Attribution Non-Commercial License (http://creativecommons.org/licenses/by -nc/3.0/) which permits unrestricted non-commercial use, distribution and reproduction in any medium, provided the original work is properly cited. 
$X$. The group $T$, with identity $e$, is assumed to be topologically discrete and remain fixed throughout this paper, so we may write $X$ instead of $(X, T)$.

A point transitive flow, $\left(X, x_{0}\right)$ consists of a flow $X$ with a distinguished point $x_{0}$ which has dense orbit.

A homomorphism of flows is a continuous, equivariant map. A homomorphism whose domain is point transitive is determined by its value at a single point. A one-one homomorphism of $X$ onto $X$ is called an automorphism of $X$. We denote the group of automorphisms of $X$ by $A(X)$.

A flow is said to be minimal if every point has dense orbit. Minimal flows are also referred to as minimal sets. $M$ is said to be a universal minimal set if it is a minimal set such that every minimal set is a homomorphic image of $M$. A homomorphism whose range is minimal is always onto.

The points $x, x^{\prime} \in X$ are said to be proximal if there exists a net $\left(t_{i}\right)$ in $T$ such that $\lim x t_{i}=\lim x^{\prime} t_{i}$. The points $x, x^{\prime} \in X$ are said to be distal if either $x=x^{\prime}$ or $x$ and $x^{\prime}$ are not proximal. Thus if $x$ and $x^{\prime}$ are both proximal and distal, they must be equal. The set of all proximal pairs in $X$ will be denoted $P(X, T)$ or simply $P . X$ is said to be proximal if $P=X \times X$. Given any point $x \in X$, we define $P(x)=\left\{x^{\prime} \in X \mid\left(x, x^{\prime}\right) \in P\right\}$.

A homomorphism $\pi: X \rightarrow Y$ is said to be proximal (resp. distal) if whenever $x, x^{\prime} \in \pi^{-1}(y)$ then $x$ and $x^{\prime}$ are proximal (resp. distal).

Given a flow $(X, T)$, we may regard $T$ as a set of self-homeomorphisms of $X$. We define $E(X)$, the enveloping semigroup of $X$ to be the closure of $T$ in $X^{X}$, taken with the product topology. $E(X)$ is at once a flow and a sub-semigroup of $X^{X}$. The minimal right ideals of $E(X)$, considered as a semigroup, coincide with the minimal sets of $E(X)$.

If $E$ is some enveloping semigroup, and there exists a homomorphism $\theta:(E, e) \rightarrow(E(X), e)$ we say that $E$ is an enveloping semigroup for $X$. If such a homomorphism exists, it must be unique, and, given $x \in X$ and $p \in E$ we may write $x p$ to mean $x \theta(p)$ unambiguously.

Lemma 2.1. ([2]) Let $E$ be an enveloping semigroup for $X$ and let $I$ be a minimal right ideal in $E$. The following are true :

(1) The set $J(I)$ of idempotent elements in $I$ is non-empty.

(2) $u p=p$ whenever $p \in I$ and $u \in J(I)$.

(3) $I u$ is a group with identity $u$ for each $u \in J(I)$. 
(4) Given $x \in X$, the following conditions are equivalent :

(a) $x$ is an almost periodic point.

(b) $\overline{x T}=x I$.

(c) $x=x u$ for some $u \in J(I)$.

Lemma 2.2. ([4]) Let $E$ be an enveloping semigroup for $X$, Then for any points $x, x^{\prime} \in X$, (a) and (b) are equivalent :

(a) $\left(x, x^{\prime}\right) \in P(X, T)$.

(b) There exists a minimal right ideal $I$ in $E$ such that $x p=x^{\prime} p$ for every $p \in I$.

Moreover, if $X$ is minimal, (a) and (b) are equivalent to :

(c) There exists $u \in J(I)$ such that $x^{\prime}=x u$.

Lemma 2.3. ([5]) If $(X, x)$ and $(Y, y)$ are point transitive flows, and $E$ is an enveloping semigroup for $X$ and $Y$, there exists a (unique) homomorphism $\zeta:(X, x) \rightarrow(Y, y)$ if and only if $x p=x q$ for $p, q \in E$ implies yp $=y q$.

Regular minimal sets were first studied by Auslander in [1]. A minimal set is said to be regular if it's isomorphic to a minimal right ideal in some enveloping semigroup.

Regular homomorphisms can be defined by extending this notion to homomorphisms with minimal range as shown in Definition 2.4 and Lemma 2.5 below.

Definition 2.4. ([5]) We say that a homomorphism $\psi: Z \rightarrow Y, Z$ and $Y$ minimal, is regular with respect to $\pi: X \rightarrow Y$ if, given any pair of homomorphisms $\gamma: M \rightarrow X$ and $\delta: M \rightarrow Z$ with $\pi \circ \gamma=\psi \circ \delta$, there exists a homomorphism $\theta: Z \rightarrow X$ with $\theta \circ \delta=\gamma$ and $\pi \circ \theta=\psi$.

Lemma 2.5. ([5]) Given a homomorphism $\pi: X \rightarrow Y$ with $X$ and $Y$ minimal, the following statements are equivalent :

(a) $\pi$ is regular.

(b) $\pi$ is regular with respect to itself.

(c) For any two points $x, x^{\prime} \in X$ with $\pi(x)=\pi\left(x^{\prime}\right)$ there exists $\xi \in$ $A(X)$ such that $\left(\xi(x), x^{\prime}\right) \in P(X, T)$ and $\pi \circ \xi=\pi$. 


\section{Some results on homomorphisms of pointed minimal sets}

Let $\beta T$ be the Stone-Cěch compactification of $T$. Then $(\beta T, e)$ is a universal point transitive flow. It is also clear that $\beta T$ is an enveloping semigroup for $X$, whenever $X$ is a flow with acting group $T$. Now let $M$ be a fixed minimal right ideal in $\beta T$. We choose a distinguished idempotent $u$ in $M$ and let $\mathcal{G}$ denote the group $M u$. Given a minimal flow $X$, we choose a point $x_{0} \in X u=\{x u \mid x \in X\}=\{x \in X \mid x u=x\}$.

Under the canonical map $\phi:(\beta T, e) \rightarrow\left(X, x_{0}\right), M$ is mapped onto $X$ and $u$ onto $x_{0}$. Thus $(M, u)$ is a universal minimal pointed flow.

Let $\left(X, x_{0}\right)$ be pointed minimal set and we define the Ellis group $\mathcal{G}\left(X, x_{0}\right)$ and the quasi-Ellis group $\mathcal{S}\left(X, x_{0}\right)$ as follows :

$$
\begin{gathered}
\mathcal{G}\left(X, x_{0}\right)=\left\{\alpha \in \mathcal{G} \mid x_{0} \alpha=x_{0}\right\}([4]) \\
\mathcal{S}\left(X, x_{0}\right)=\left\{\alpha \in \mathcal{G} \mid h\left(x_{0}\right) \alpha=x_{0} \text { for some } \mathrm{h} \in \mathrm{A}(\mathrm{X})\right\} .
\end{gathered}
$$

Clearly $\mathcal{G}\left(X, x_{0}\right) \subset \mathcal{S}\left(X, x_{0}\right)$, and $\mathcal{G}\left(X, x_{0}\right)$ is a subgroup of $\mathcal{G}$.

Theorem 3.1. Let $\left(X, x_{0}\right)$ be a pointed minimal set. Then the following statements are true :

(1) $\mathcal{S}\left(X, x_{0}\right)$ is a subgroup of $\mathcal{G}$.

(2) $\mathcal{G}\left(X, x_{0}\right)$ is a normal subgroup of $\mathcal{S}\left(X, x_{0}\right)$.

Proof. (1) To see that $\mathcal{S}\left(X, x_{0}\right)$ is a subgroup of $\mathcal{G}$, let $\alpha_{1}, \alpha_{2} \in$ $\mathcal{S}\left(X, x_{0}\right)$. Then there exist $h_{1}, h_{2} \in A(X)$ such that $h_{1}\left(x_{0}\right) \alpha_{1}=x_{0}$ and $h_{2}\left(x_{0}\right) \alpha_{2}=x_{0}$ and hence $h_{2}^{-1} \circ h_{1}\left(x_{0}\right) \alpha_{1} \alpha_{2}^{-1}=h_{2}^{-1}\left(h_{1}\left(x_{0}\right) \alpha_{1}\right) \alpha_{2}^{-1}=$ $h_{2}^{-1}\left(x_{0}\right) \alpha_{2}^{-1}=h_{2}^{-1}\left(h_{2}\left(x_{0}\right) \alpha_{2}\right) \alpha_{2}^{-1}=x_{0}$. Since $\alpha_{1} \alpha_{2}^{-1} \in \mathcal{G}$ and $h_{2}^{-1} \circ$ $h_{1} \in A(X)$, it follows that $\alpha_{1} \alpha_{2}^{-1} \in \mathcal{S}\left(X, x_{0}\right)$.

(2) Let $\alpha \in \mathcal{G}\left(X, x_{0}\right)$ and let $\beta \in \mathcal{S}\left(X, x_{0}\right)$. Then $x_{0} \alpha=x_{0}$ and $h\left(x_{0}\right) \beta=x_{0}$ for some $h \in A(X)$ and hence $x_{0}\left(\beta^{-1} \alpha \beta\right)=\left(h\left(x_{0}\right) \beta\right) \beta^{-1} \alpha \beta=$ $h\left(x_{0} \alpha\right) \beta=h\left(x_{0}\right) \beta=x_{0}$. Since $\mathcal{G}$ is a group, it follows that $\beta^{-1} \alpha \beta \in$ $\mathcal{G}\left(X, x_{0}\right)$. This proves that $\mathcal{G}\left(X, x_{0}\right)$ is a normal subgroup of $\mathcal{S}\left(X, x_{0}\right)$.

Theorem 3.2. ([4]) Let $\pi:\left(X, x_{0}\right) \rightarrow\left(Y, y_{0}\right)$ be a homomorphism of pointed minimal sets. Then the following statements are true:

(1) $\mathcal{G}\left(X, x_{0}\right) \subset \mathcal{G}\left(Y, y_{0}\right)$

(2) $\mathcal{G}\left(X, x_{0}\right)=\mathcal{G}\left(Y, y_{0}\right)$ if and only if $\pi$ is proximal.

(3) $\pi$ is distal if and only if for every $y \in Y$ and $p \in M$ such that $y_{0} p=y, \pi^{-1}(y)=x_{0} \mathcal{G}\left(Y, y_{0}\right) p$. 
REMARK 3.3. The following statements are true:

(1) Let $\pi:\left(X, x_{0}\right) \rightarrow\left(Y, y_{0}\right)$ be a proximal homomorphism of pointed minimal sets and suppose $\pi$ is a constant fuction. Then $\mathcal{G}\left(X, x_{0}\right)=$ $\mathcal{S}\left(X, x_{0}\right)$. To see the proof, note that if $X$ is proximal and minimal, then the only homomorphism $\eta: X \rightarrow X$ is the identity (see [3]).

(2) Suppose $\pi:\left(X, x_{0}\right) \rightarrow\left(Y, y_{0}\right)$ is an isomorphism of pointed minimal sets. Then $\mathcal{S}\left(X, x_{0}\right)=\mathcal{S}\left(Y, y_{0}\right)$ as the following proof shows. For any $\alpha \in \mathcal{S}\left(X, x_{0}\right)$, there exists $h \in A(X)$ such that $h\left(x_{0}\right) \alpha=x_{0}$. Since $\pi h \pi^{-1}\left(y_{0}\right) \alpha=y_{0}$ and $\pi h \pi^{-1} \in A(Y)$, it follows that $\mathcal{S}\left(X, x_{0}\right) \subset \mathcal{S}\left(Y, y_{0}\right)$. Also $\pi^{-1}:\left(Y, y_{0}\right) \rightarrow\left(X, x_{0}\right)$ is an isomorphism.

Thus $\mathcal{S}\left(Y, y_{0}\right) \subset \mathcal{S}\left(X, x_{0}\right)$.

TheOrEm 3.4. Let $\pi:\left(X, x_{0}\right) \rightarrow\left(Y, y_{0}\right)$ be a homomorphism of pointed minimal sets. Then $\pi$ is regular if and only if $\mathcal{G}\left(Y, y_{0}\right) \subset$ $\mathcal{S}\left(X, x_{0}\right)$.

Proof. Suppose that $\pi:\left(X, x_{0}\right) \rightarrow\left(Y, y_{0}\right)$ is a regular homomorphism of minimal sets and that $\alpha \in \mathcal{G}\left(Y, y_{0}\right)$. Let $\gamma:(M, u) \rightarrow\left(X, x_{0}\right)$ and $\delta:(M, u) \rightarrow\left(Y, y_{0}\right)$ be homomorphisms with $\pi \circ \gamma=\delta$. The minimality of $M$ allows us to define $\rho: M \rightarrow M$ by $\rho(u)=\alpha$. Also, $u p=u q$ implies $\alpha p=\alpha q$ for $p, q \in M$. This shows that $\rho:(M, u) \rightarrow(M, \alpha)$ is a unique homomorphism by Lemma 2.3. Since $\alpha, u \in \mathcal{G} \subset M$ and $\mathcal{G}$ is a group, it follows that $\rho \in A(M)$. Also $\pi \circ \gamma \circ \rho(u)=\pi \circ \gamma(\alpha)=\pi(\gamma(u) \alpha)=$ $\pi\left(x_{0}\right) \alpha=y_{0} \alpha=y_{0}=\pi\left(x_{0}\right)=\pi \circ \gamma(u)$, and thus $\pi \circ(\gamma \circ \rho)=\pi \circ \gamma$. Since $\pi$ is regular, we have from Lemma 2.5 that there exists $h \in A(X)$ such that $h \circ(\gamma \circ \rho)=\gamma$ and $\pi \circ h=\pi$. Hence $h\left(x_{0}\right) \alpha=h(\gamma(u) \alpha)=$ $h(\gamma(\alpha))=h(\gamma(\rho(u)))=h \circ \gamma \circ \rho(u)=\gamma(u)=x_{0}$ whence $\alpha \in S\left(X, x_{0}\right)$.

Conversely suppose that $\gamma: M \rightarrow X$ and $\delta: M \rightarrow X$ are homomorphisms with $\pi \circ \gamma=\pi \circ \delta$. Then there exists $\eta \in A(M)$ such that $\delta=\gamma \circ \eta$ by Lemma 2.3.1 in [5]. Let $\eta(u)=\alpha$. Then $y_{0}=\pi\left(x_{0}\right) \alpha=$ $\pi(\gamma(u)) \alpha=\pi \circ \gamma \circ \eta(u)=\pi \circ \delta(u)=\pi \circ \gamma(u)=\pi\left(x_{0}\right)=y_{0}$ and so $\alpha \in \mathcal{G}\left(Y, y_{0}\right)$. Since $\mathcal{G}\left(Y, y_{0}\right) \subset \mathcal{S}\left(X, x_{0}\right)$, it follows that there exists $h \in A(X)$ with $h\left(x_{0}\right) \alpha=x_{0}$. Therefore $h \circ \delta(u)=h \circ \gamma \circ \eta(u)=$ $h \circ \gamma(\alpha)=h(\gamma(u) \alpha)=h\left(x_{0}\right) \alpha=x_{0}=\gamma(u)$ from which it follows that $h \circ \delta=\gamma$. For any $x \in X$, pick $m \in M$ with $\delta(m)=x$. Then $\pi \circ h(x)=\pi \circ h \circ \delta(m)=\pi \circ \gamma(m)=\pi \circ \delta(m)=\pi(x)$ whence $\pi \circ h=\pi$. This shows that $\pi$ is regular with respect to itself. Consequently $\pi$ is regular. 
Corollary 3.5. Let $\pi:\left(X, x_{0}\right) \rightarrow\left(Y, y_{0}\right)$ be a homomorphism of pointed minimal sets. If $\pi$ is regular, then $\mathcal{G}\left(X, x_{0}\right)$ is a normal subgroup of $\mathcal{G}\left(Y, y_{0}\right)$.

Proof. Let $\alpha \in \mathcal{G}\left(X, x_{0}\right)$ and let $\beta \in \mathcal{G}\left(Y, y_{0}\right)$. Since $\pi$ is regular, it follows from Theorem 3.4 that $\mathcal{G}\left(Y, y_{0}\right) \subset \mathcal{S}\left(X, x_{0}\right)$. Hence $x_{0} \alpha=x_{0}$ and $h\left(x_{0}\right) \beta=x_{0}$ for some $h \in A(X)$. Then $x_{0}\left(\beta^{-1} \alpha \beta\right)=\left(h\left(x_{0}\right) \beta\right) \beta^{-1} \alpha \beta=$ $h\left(x_{0} \alpha\right) \beta=h\left(x_{0}\right) \beta=x_{0}$. Thus $\beta^{-1} \alpha \beta \in \mathcal{G}\left(X, x_{0}\right)$. This implies that $\mathcal{G}\left(X, x_{0}\right)$ is a normal subgroup of $\mathcal{G}\left(Y, y_{0}\right)$.

TheOrem 3.6. Let $\pi:\left(X, x_{0}\right) \rightarrow\left(Y, y_{0}\right)$ be a homomorphism of pointed minimal sets and let $y \in Y$ and $p \in M$ such that $y_{0} p=y$. If $x_{0} \mathcal{G}\left(Y, y_{0}\right) p \subset x_{0} J(M)$, then $y \in P\left(y_{0}\right)$.

Proof. Let $y \in Y$ and $p \in M$ such that $y_{0} p=y$. For each $\alpha \in \mathcal{G}\left(Y, y_{0}\right)$, there exists $v \in J(M)$ such that $x_{0} \alpha p=x_{0} v$. Since $\alpha \in \mathcal{G}\left(Y, y_{0}\right)$, it follows that $y=y_{0} p=y_{0} \alpha p=\pi\left(x_{0} \alpha p\right)=\pi\left(x_{0} v\right)=y_{0} v$. Hence $y \in P\left(y_{0}\right)$.

Theorem 3.7. ([4]) Suppose that $\pi:\left(X, x_{0}\right) \rightarrow\left(Y, y_{0}\right)$ is a homomorphism of pointed minimal sets and $y \in Y$ and that $\pi$ is proximal. Then $\pi^{-1}(y) \subset x J(M)$ for all $x \in \pi^{-1}(y)$.

Theorem 3.8. Suppose that $\pi: X \rightarrow Y$ is a homomorphism of minimal sets and $y \in Y$ and that $\pi$ is regular. Then, for each $x^{\prime} \in \pi^{-1}(y)$, there exists $\xi \in A(X)$ such that $\xi\left(x^{\prime}\right) \in x J(M)$ for all $x \in \pi^{-1}(y)$.

Proof. Let $x, x^{\prime} \in \pi^{-1}(y)$. Since $\pi$ is regular, it follows that there exists $h \in A(X)$ with $\left(h(x), x^{\prime}\right) \in P(X, T)$ and by Lemma 2.2 there exists a minimal right ideal $I$ such that $x^{\prime}=h(x) v^{\prime}$ for some $v^{\prime} \in J(I)$. Then there exists a unique idempotent $v \in J(M)$ with $v v^{\prime}=v$ and $v^{\prime} v=v^{\prime}$ by [2, Proposition 3.6] and hence $\pi\left(h(x) v^{\prime}\right)=\pi\left(x^{\prime}\right)=y$ and $\pi(x v)=y v=\pi\left(h(x) v^{\prime}\right) v=\pi\left(h(x) v^{\prime} v\right)=\pi\left(h(x) v^{\prime}\right)=y$. Since $\pi$ is regular, we have from Lemma 2.5 that there exists $\xi \in A(X)$ such that $\left(\xi\left(h(x) v^{\prime}\right), x v\right) \in P(X, T)$. But $\xi(h(x)) v^{\prime} v=\xi(h(x)) v^{\prime}$ and $x v v=x v$, and by Lemma $2.1 \xi(h(x)) v^{\prime}$ and $x v$ are also almost periodic points. Therefore $\xi\left(x^{\prime}\right)=\xi\left(h(x) v^{\prime}\right)=x v \in x J(M)$ by [2, Remark 5.11].

Theorem 3.9. ([4]) Let $\pi:\left(X, x_{0}\right) \rightarrow\left(Y, y_{0}\right)$ and $\tau:\left(Z, z_{0}\right) \rightarrow\left(Y, y_{0}\right)$ be two distal homomorphisms of pointed minimal sets. There exists a homomorphism $\mu:\left(Z, z_{0}\right) \rightarrow\left(X, x_{0}\right)$ if and only if $\mathcal{G}\left(Z, z_{0}\right) \subset \mathcal{G}\left(X, x_{0}\right)$. 


\section{References}

[1] J. Auslander, Regular minimal sets 1, Trans. Amer. Math. Soc. 123 (1966), 469479.

[2] R. Ellis, Lectures on topological dynamics, Benjamin, New York, 1969.

[3] S. Glasner, Compressibility properties in topological dynamics, Amer. J. Math. 97 (1975), 148-171.

[4] , Proximal flows, Springer-Verlag, New York, 1975.

[5] P. S. Shoenfeld, Regular homomorphisms of minimal sets, Doctoral Dissertation, Univ. of Maryland (1974).

Department of Mathematics

Kwangwoon University

Seoul 139-701, Korea

E-mail: songhs@kw.ac.kr 